\title{
TRATAMIENTOS DE AGUAS INDUSTRIALES CON METALES PESADOS A TRAVÉS DE ZEOLITAS Y SISTEMAS DE BIORREMEDIACIÓN. REVISIÓN DEL ESTADO DE LA CUESTIÓN
}

\author{
(Industrial water treatment with heavy metals through zeolites and bioremediation systems \\ with aquatic plants especially Eichhornia crassipes. State of art review)
}

Uriel Fernando Carreño Sayago

Facultad de Ingeniería, Fundación Universitaria los Libertadores. Grupo de investigación Bio-ingenio. ufcarrenos@libertadores.edu.co

(Recibido el 9 de julio de 2014 y aceptado el 30 de octubre de 2014)

\begin{abstract}
Resumen:
En la presente revisión se exploran diferentes oportunidades de utilizar un material natural barato para la remoción y retención de metales pesados de aguas contaminadas por los residuos de diferentes procesos. Se abordarán dos sistemas investigativos: el primero a través de un material conocido como zeolita $\mathrm{o}$, de manera más general, aluminosilicatos porosos, que podrá ser sintetizado o extraído de las minas de arcillas y minerales, siendo usado en su estado natural o tras procesos de modificación y dopaje; y el otro sistema es la biorremediacion a través de algas, algunas bacterias y sobre todo plantas acuáticas como el Eichhornia crassipes (buchón de agua). Se evaluará la viabilidad de juntar estos dos tipos de tratamientos complementando el uno con el otro. También se revisarán investigaciones sobre la viabilidad de Eichhornia crassipes (buchón de agua) como materia prima para biocombustibles.
\end{abstract}

Palabras clave: zeolitas, Eichhornia crassipes (buchón de agua), biorremediación.

\section{INTRODUCCIÓN}

El mundo está enfrentando una crisis por la falta de agua. Dicha escasez es una consecuencia del rápido desarrollo de las industrias y la gran cantidad de agua residual de

\begin{abstract}
:
In this review we explore different opportunities to use a cheap natural material for removing and retention of heavy metals from polluted waters by waste of different processes. Two research systems will be addressed: the first through a material known as zeolite or more generally porous aluminosilicates, which may be synthesized or extracted from the mines of clays and minerals, being used in its natural state or after modification processes and doping. The other mechanism is bioremediation through algae, some bacteria and especially aquatic plants such as Eichhornia crassipes (water hyacinth). We will evaluate the viability of joining these two types complementing each other. Investigations into the feasibility of Eichhornia crassipes (water hyacinth) as feedstock for biofuels are also reviewed.
\end{abstract}

Keywords: Zeolites, Eichhornia crassipes (water hyacinth), bioremediation. procesos industriales que se descarga a los ríos y demás sistemas hídricos. Estas aguas residuales suelen contener una gran variedad de contaminantes, muchos en forma de iones catiónicos y aniónicos, aceites y grasas, y demás residuos orgánicos con efectos nocivos y venenosos sobre los ecosistemas. Generalmente, la remoción de 
estos contaminantes requiere de tecnologías efectivas, por lo que en las últimas décadas se han desarrollado técnicas de limpieza que tratan esta problemática. Actualmente se cree que la absorción es el mecanismo más simple y efectivo para el tratamiento de las aguas residuales, donde el éxito de esta depende del desarrollo de absorbentes eficientes (Wang \& Peng, 2010; Epstein, 1998; Gómez \& Pinzón, 2012).

En esta revisión se explorará la oportunidad de utilizar dos tipos de materiales naturales y baratos para la remoción y retención de metales pesados de aguas contaminadas por los residuos del proceso industrial. Específicamente se revisará sobre dos posibilidades: un primer material de origen mineral y un segundo material de origen vegetal. El material de origen mineral se trata de zeolitas y mesoporosos, los cuales podrán ser sintetizados o extraídos de las minas de arcillas y minerales en Colombia, y usados en su estado natural o tras procesos de modificación y dopaje. El segundo material, de origen vegetal, como las macrofitas especialmente el Eichhornia crassipes (buchón de agua), (Chuang et al., 2012).

Las zeolitas son materiales adecuados para el tratamiento de agua con metales pesados, dada su gran capacidad de intercambio iónico, así como su gran afinidad por este tipo de metales. Este material presenta una red tridimensional que consiste en unidades tetrahédricas de óxidos de silicio y aluminio unidas por un átomo de oxígeno compartido. El reemplazo isomorfo de $\mathrm{Si} 4+$ por $\mathrm{Al} 3+$ resulta en una carga negativa global que es compensada por una base o cationes metálicos basificados del medio (Covarrubias et al. 2008; Margeta et al. 2013; Wang \& Peng, 2010).

Estos materiales de origen natural son minados de distintos depósitos a lo largo del mundo entero. Así mismo, las zeolitas pueden ser producidas sintéticamente con propiedades especiales para aplicaciones específicas. Las zeolitas naturales, en particular la clinoptolita, han sido estudiadas extensivamente para la remoción de metales pesados de aguas residuales, debido a su gran disponibilidad y bajo costo (Margeta et al., 2013).

Por otra parte, los estudios en zeolitas sintéticas tienden a enfocarse en la determinación de parámetros termodinámicos de procesos de catálisis, y generalmente no han sido consideradas para aplicaciones medioambientales (Wang \& Peng, 2010).
Además de las zeolitas, se examinará la capacidad de diferentes agentes biológicos para la biorremediación, especialmente la Eichhornia crassipes (buchón de agua), (Higuera et al., 2008). El objeto de este trabajo es establecer el estado de la cuestión sobre la remoción de metales pesados utilizando un material biológico, siendo una forma alterna a las convencionales, cuyo montaje es de bajo costo.

\section{SISTEMAS DE TRATAMIENTO DE AGUAS CON ZEOLITAS}

Las zeolitas son materiales adecuados para el tratamiento de agua (Margeta et al., 2013; Wang \& Peng, 2010) con metales pesados, dada su gran capacidad de intercambio catiónico, así como su gran afinidad por este tipo de metales (Hui et al., 2005; Ríos et al., 2013). Las zeolitas naturales son minerales de aluminosilicatos hidratados con una estructura porosa y propiedades fisicoquímicas valiosas, además del intercambio de cationes, entre las que se cuentan el cribado molecular, la catálisis y la absorción (Ríos et al., 2013); (Pitcher et al., 2004). De manera simple, se trata de una red tridimensional que consiste en unidades tetrahedrales de sílice y alúmina unidas a un átomo de oxígeno compartido. El reemplazo isomorfo de Si4+ por Al3+ resulta en una carga negativa global que es compensada con diversos cataiones, otorgando a este material propiedades adicionales a las estructurales (Wang \& Peng, 2010).

Se han llevado a cabo diversos estudios sobre su utilización para la absorción y reducción de Cr (VI) en distintos ambientes (acuosos, etc.), (Covarrubias et al., 2008; Asgari et al., 2013). (Yubin et al, 2015).

En el tratamiento de cromo utilizado en las curtiembres, se han hecho estudios relacionados con las aplicaciones de materiales zeolíticos en la eliminación de estos iones (Covarrubias et al., 2008), los cuales han demostrado que al poner en contacto zeolitas de distinta estructura (4A, 13X y mordenita), con una solución preparada con sales de cromo $\mathrm{Cr}$ (III) y $\mathrm{Cr}$ (VI), siempre había una absorción y retención preferencial del $\mathrm{Cr}$ (VI) respecto al $\mathrm{Cr}$ (III) en la estructura de las mismas. Dicha capacidad de retención se incrementaba cuando las zeolitas eran modificadas con surfactantes.

En los últimos años se han probado distintos tipos de modificación de las zeolitas, siendo la más sobresaliente para la aplicación en aguas residuales y remoción de $\mathrm{Cr}$ 
(VI), el dopaje de estas con Fe. Guocheng et al. (2014) y Du et al. (2012), demostraron que al modificar zeolita natural con $\mathrm{Fe}$ (III), la adsorción de $\mathrm{Cr}$ (VI) aumenta, así como la concentración de equilibrio de Cr (VI). A partir de este resultado se demostró que la zeolita natural, modificada con $\mathrm{Fe}$, puede servir como medio, nada o poco costoso, para la remoción del $\mathrm{Cr}(\mathrm{VI})$ del agua.

Wanga y Peng (2012) concluyeron que las zeolitas naturales son abundantes y de bajo costo, además establecieron que la zeolita pretratada es un buen adsorbente de arsénico, con más de $90 \%$ de adsorción. Se puede decir que la incorporación de óxidos de magnesio sobre la zeolita es un factor muy significativo para obtener resultados favorables en la adsorción de arsénico.
Ríos et al. (2013) determinaron que el Ca2+ es el metal que presenta mayor capacidad de intercambio con respecto a los demás cationes intercambiables presentes en la estructura de la zeolita, y también que el aumento en la concentración de iones $\mathrm{Na}+$ en solución favorece el intercambio de dichos cationes. El proceso de adsorción de Fe y Mn se ve favorecido por el empleo de zeolita acondicionada con las capas de $\mathrm{Fe} 2 \mathrm{O} 3$ y $\mathrm{MnO} 2$ a partir de las sales $\mathrm{FeCl} 3$ y $\mathrm{MnSO} 4$, respectivamente. También en el estudio de Wang y Peng (2010) se comprobó el poder de algunas zeolitas en la depuración de algunos compuestos orgánicos de solución acuosa.

A continuación se describen investigaciones en que se han utilizado las zeolitas como agentes de tratamiento de aguas industriales contaminadas con metales pesados. Estos trabajos se realizaron a escala piloto.

Tabla 1. Trabajos sobre zeolitas en tratamiento de metales pesados.

\begin{tabular}{|c|c|c|}
\hline Autores & Metal tratado & Tipo de tratamiento \\
\hline $\begin{array}{l}\text { Mejía } \\
(\mathbf{2 0 1 0})\end{array} \quad$ et al. & & $\begin{array}{l}\text { Construyeron un reactor a escala piloto, donde se puso en contacto el } \\
\text { agua contaminada con (Ar) por más de } 5 \text { días. }\end{array}$ \\
\hline Ríos et al. (2013) & $\begin{array}{l}\text { Hierro }(\mathrm{Fe}) \text { Man- } \\
\text { ganeso(Mn) }\end{array}$ & $\begin{array}{l}\text { Desarrollaron una metodología donde se puso en contacto el agua con- } \\
\text { taminada con }(\mathrm{Fe}) \text { y }(\mathrm{Mn}) \text { por más de } 10 \text { días, arrojando unas remocio- } \\
\text { nes por encima del } 85 \% \text {. }\end{array}$ \\
\hline $\begin{array}{l}\text { Magriotis et al. } \\
\text { (2014). }\end{array}$ & & $\begin{array}{l}\text { Utilizaron una concentración inicial de eteramina de } 200(\mathrm{mg} / \mathrm{L}) \text {. La } \\
\text { adsorción era favorable a un } \mathrm{pH} \text { de } 10,0 \text {. Se alcanzó el equilibrio de } \\
\text { adsorción en } 30 \text { min y remociones por encima del } 96 \% \text {. }\end{array}$ \\
\hline $\begin{array}{l}\text { Chutia et al. } \\
\text { (2008) }\end{array}$ & & $\begin{array}{l}\text { Desarrollaron una metodología donde se pusieron en contacto las zeo- } \\
\text { litas con diferentes concentraciones de una solución acuosa de (Ar). } \\
\text { Arrojando resultados favorables de más del } 80 \% \text { en remoción. }\end{array}$ \\
\hline $\begin{array}{l}\text { Leyva } \\
(2008)\end{array}$ & & $\begin{array}{l}\text { Diseñaron y construyeron un reactor por lotes, donde se puso en con- } \\
\text { tacto el cromo con las zeolitas. }\end{array}$ \\
\hline $\begin{array}{l}\text { Acevedo et al. } \\
\text { (2011) }\end{array}$ & Mixto & $\begin{array}{l}\text { Diseñaron y construyeron un primer filtro de flujo descendente, conec- } \\
\text { tado al segundo de flujo ascendente, ambos con un diámetro de } 10.5 \\
\mathrm{~cm} \text {, una altura de } 60 \mathrm{~cm} \text {, y altura de empaque de la zeolita } 50 \mathrm{~cm} \text {. }\end{array}$ \\
\hline $\begin{array}{l}\text { Ozdemir et al. } \\
\text { (2012) }\end{array}$ & Color & $\begin{array}{l}\text { Construyeron un reactor de columna de lecho fijo y la superficie de la } \\
\text { zeolita natural, experimentos con contacto directo. Se obtuvieron re- } \\
\text { mociones por encima del } 90 \% \text {. }\end{array}$ \\
\hline $\begin{array}{l}\text { Fernández et al. } \\
\text { (2008) }\end{array}$ & Aguas residuales & $\begin{array}{l}\text { Diseñaron y construyeron un reactor de lecho fluidizado anaeróbico } \\
\text { con zeolita natural como material de apoyo en el tratamiento de alta } \\
\text { resistencia de aguas residuales, se obtuvieron remociones por encima } \\
\text { del } 90 \% \text {. }\end{array}$ \\
\hline $\begin{array}{l}\text { Katsou et al. } \\
\text { (2011) }\end{array}$ & $\begin{array}{l}\text { Zinc }(\mathrm{Zn}) \text { y Plomo } \\
(\mathrm{Pb})\end{array}$ & $\begin{array}{l}\text { Prepararon unas soluciones acuosas en contacto con las zeolitas. Se } \\
\text { obtuvieron remociones por encima del } 80 \% \text {. }\end{array}$ \\
\hline
\end{tabular}




\begin{tabular}{|c|c|c|}
\hline Autores & Metal tratado & Tipo de tratamiento \\
\hline $\begin{array}{l}\text { Qiu \& Zheng, } \\
\text { (2009) }\end{array}$ & $\begin{array}{l}\text { Niquel (Ni) Cobal- } \\
\text { to }(\mathrm{Co})\end{array}$ & $\begin{array}{l}\text { Diseñaron y construyeron un reactor de contacto directo zeolitas y so- } \\
\text { lución acuosa, en el que el tiempo de retención fue de un día. Se obtu- } \\
\text { vieron remociones por encima del } 60 \% \text {. }\end{array}$ \\
\hline $\begin{array}{l}\text { Guocheng et al. } \\
\text { (2014) }\end{array}$ & Cromo (Cr) & $\begin{array}{l}\text { Realizaron un estudio con zeolitas modificadas y sin modificar, se pu- } \\
\text { sieron en contacto con soluciones acuosas de diferentes metales pesa- } \\
\text { dos. }\end{array}$ \\
\hline $\begin{array}{l}\text { Kumar et al. } \\
\text { (2008) }\end{array}$ & Níquel & $\begin{array}{l}\text { Construyeron un reactor con zeolitas y carbón activado, obteniendo } \\
\text { remociones por encima del } 80 \% \text {. }\end{array}$ \\
\hline $\begin{array}{l}\text { Blanco } \\
\text { (2014) }\end{array}$ & Plomo $(\mathrm{Pb})$ & $\begin{array}{l}\text { Construyeron un proceso terciario de aguas residuales. Reactor a escala } \\
\text { piloto, donde se trataban las aguas contaminadas con plomo, remocio- } \\
\text { nes por encima del } 70 \% \text {. }\end{array}$ \\
\hline
\end{tabular}

\section{SISTEMAS DE TRATAMIENTO DE AGUAS CON DIFERENTES AGENTES BIOLÓGICOS}

En esta sección se muestran diferentes tratamientos con agentes biológicos.

Un sistema de tratamiento a través de la biorremediación es una tendencia a nivel mundial, debido a sus bajos costos en la implementación y sobre todo a la capacidad de las algas como agente biológico importante, las cuales se encuentran fácilmente en cualquier sistema acuático y son eficientes a la hora de remover metales pesados presentes en las aguas, ya que absorben estos metales incorporándolos en su estructura biológica. Por ejemplo, Veranes et al. (2009) estudiaron las algas Scenedesmus obliquus, y demostraron el poder de este microorganismo a la hora de remover el cromo. También Higuera et al. (2005) utilizaron el alga parda Sargassum sp y removieron el $85 \%$ de cromo.

Sari y Tusen (2008) encontraron la cinética y el crecimiento de un cultivo de algas para el tratamiento de efluentes cargados de cromo provenientes de curtiembres. Han et al. (2008) determinaron el grado de remoción de las algas Chlorella miniata a escala piloto. Pellón et al. (2011) diseñaron un biorreactor para la remoción de metales pesados y utilizaron diferentes tipos de algas.

Las algas son microrganismos altamente eficientes a la hora de remover metales pesados, pero también las bacterias son capaces de ejercer esta labor importante en el tratamiento de las aguas industriales. Rodríguez (2013), quien estudió la cepa de Strepcoccus sp y Kingella kingae, obtuvo resultados que prueban la factibilidad de utilizar tecnología innovadora, barata y eficiente, basada en herramientas biotecnológicas, para depurar aguas residuales industriales altamente contaminadas en cromo y en otros metales pesados.

En diferentes investigaciones también se ha encontrado que las plantas son agentes bioacumuladores de metales pesados, como en el caso de las hojas de café en el estudio de Higuera et al. (2008), quienes establecieron una metodología práctica a la hora de retener metales pesados presentes en las aguas, diseñando e implementando un filtro rápido de arena, el cual se compone de una capa de grava y unas capas trituradas de hojas de café que se situaron en tres posiciones diferentes (inferior, media y superior). Ellos concluyeron que este sistema de tratamiento a escala piloto es eficiente, puesto que remueve el $90 \%$ de los metales pesados.

Las plantas acuáticas, como la Lemna minor (lenteja de agua), son especialmente utilizadas en sistemas de tratamiento no convencionales en la remoción de metales pesados, hecho que se documentó en el estudio de Arenas et al. (2011), donde se hizo una investigación a escala piloto, estableciendo un grupo control (agua destilada + mercurio) y un grupo testigo (agua destilada + Lemna minor) y un grupo experimental (agua destilada + mercurio + Lemna minor) por un tiempo de retención de 22 días con remociones de más de $50 \%$.

Pero de las plantas acuáticas encontradas en la literatura actual se debe hacer especial referencia a la Eichhornia crassipes (buchón de agua), debido a su alta capacidad para la retención de metales pesados presentes en el agua y para la generación de energía (Gopal et al., 1987). Esta planta acuática es invasora de ecosistemas acuáticos y se 
encuentra en grandes cantidades en humedales, lagunas, ríos, etc. (Epstein, 1998; Gómez \& Pinzón, 2012).

En la remoción de metales pesados se ha demostrado su alta eficiencia, como en el caso del estudio de Vázquez (2010), en el cual se hizo una investigación con tintes industriales, donde se utilizaron tres recipientes con capacidad para 20 litros cada uno, y se inoculó la Eichhornia crassipes (buchón de agua), durante ocho días, obteniendo remociones por encima del $90 \%$. Vázquez concluyó que esta planta acuática es altamente eficiente a la hora de remover metales pesados. También Velarde et al (2013) realizó una simulación de un efluente de una curtiembre con $\mathrm{K} 2 \mathrm{Cr} 2 \mathrm{O} 7$ a concentraciones de 10ppm, $25 \mathrm{ppm}$ y $50 \mathrm{ppm}$ por un período de nueve semanas en un sistema hidropónico libre con la Eichhornia crassipes (buchón de agua), obteniendo unas remociones por encima del $80 \%$.

Uno de los primeros estudios realizados con buchón de agua fue el desarrollado por Lenka et al. (1990), quienes evaluaron estas plantas con concentraciones de mercurio acuático. Después de determinado tiempo, se muestrearon las raíces del buchón analizando la bioconcentración de mercurio a través de espectrofotometría, y los resultados indicaron que la bioconcentración de mercurio en el tejido de la raíz era tanto tiempo y dependiente de la concentración, hecho que proporcionó pruebas de que el buchón de agua es un buen absorbente de mercurio acuático.

Atehortúa y Gartner (2013) tamizaron la Eichhornia crassipes (buchón de agua) para construir un filtro biológico para el tratamiento de aguas industriales contaminadas con cromo y plomo, el tiempo de retención fue de 6 horas y se removió un $60 \%$ de estos metales. También, Chisutia y Mmari (2014); Xiaosen et al. (2013); tamizaron la Eichhornia crassipes (buchón de agua) para tratar efluentes de industrias, arrojando eficiencias por encima del $90 \%$. En el estudio de Torres (2009), se analizó la capacidad de sorción de la E. crassipes seca por medio de ensayos de flujo y se encontró que esta capacidad depende de variables como la velocidad de flujo, el pH de la solución y el tamaño de partículas.

Se han diseñado y construido diferentes sistemas de tratamiento con la Eichhornia crassipes (buchón de agua), que consistieron en colocar esta planta en aguas industriales contaminadas determinando sus remociones y estableciendo variables en el diseño como capacidad, $\mathrm{pH}$, temperatura, entre otras (Zimmels et al., 2006; Alvarado et al. 2008; Saraswat, 2010; Carrión et al., 2012).

Kasturiarachchi (2014) diseñó y construyó un sistema de tratamiento con el buchón acuático como agente principal, en tanque de fibra de vidrio, para el tratamiento de nutrientes, y concluyó que esta planta es vital para construir humedales artificiales.

Un mecanismo biológico estudiado por Tang (2015) concluyó que cuando el buchón de agua se encuentra reteniendo metales como el uranio, activa unas enzimas antioxidantes que ayudan a la retención de este metal. Martínez et al. (2013) estudiaron la cinética de absorción de la Eichcornia crassipes, evaluando diferentes ecuaciones de primer y segundo orden.

El buchón de agua, como se ha podido demostrar, es un agente interesante a la hora de remover metales, pero al ser una planta acuática muy abundante en muchos ecosistemas y su alta capacidad reproductora, también se ha investigado sobre su alta producción energética. Como en el estudio de Dhahiyat et al., (2012), donde experimentaron sobre la producción de hidrógeno a partir del buchón de agua, utilizando microflora de excremento de cerdo. Estos autores evaluaron la producción de metano e hidrógeno con diferentes concentraciones de esta planta acuática.

En la actualidad se centra mucha atención en el desarrollo de métodos para producir etanol a partir de biomasa con alto contenido de celulosa, y el buchón de agua, siendo una planta abundante, tiene las características esenciales para este propósito (Hossain et al., 2010).

El buchón de agua cumple los criterios para la producción de bioenergía, pues es permanente -debido a que hay grandes cantidades de plantas disponibles-, biodegradable y tiene un alto contenido de celulosa (Porous et al., 2012). Sin embargo, su fuerte desventaja es que tiene más del $90 \%$ de contenido de agua, lo que complica el proceso de cosecha y procesamiento. La biomasa puede ser sometida a la producción de biogás para generar energía para uso doméstico en las zonas rurales (Chuang et al., 2012).

El buchón de agua posee bajos contenidos de lignina $(10 \%)$, contiene alta cantidad de celulosa $(20 \%)$ y hemicelulosa (33\%), siendo la celulosa y la hemicelulosa 
compuestos fácilmente convertibles en azúcar fermentable, lo que resulta, por tanto, en una enorme cantidad de biomasa funcional para la industria de los biocombustibles (Poddar et al., 1991; Ikhtyar et al., 1984; Dhahiyat et al., 2012; Bolenz et al., 2010; Gutiérrez et al., 2012; Kumar \& Ghosh, 2009; Mishima et al., 2008). También Aswathy et al. (2010) estudiaron las enzimas celulasa y $\beta$-glucosidasa para la degradación del buchón de agua, el objetivo era establecer los mecanismos con las que se obtiene energía.

\section{CONCLUSIONES}

Las zeolitas son materiales adecuados para el tratamiento de agua con metales pesados, dada su gran capacidad de intercambio catiónico, así como su gran afinidad por este tipo de metales. Son económicos, fáciles de conseguir y se podrían adaptar a otros sistemas de tratamiento, como la construcción de filtros de zeolitas y biológicos.

Debido al alto crecimiento y abundancia del buchón del agua, y teniendo en cuenta que esta planta retiene en su organismo grandes contenidos de metales pesados, se propone una tecnología de fácil acceso y económica para tratar las aguas contaminadas con metales pesados.

También se encontró que de esta planta se puede obtener la suficiente energía como el biogás y el hidrógeno, siendo esta utilización viable para implementar.

La creación de unas tecnologías de tratamiento a través de zeolitas y plantas acuáticas es una opción viable en las industrias que vierten a los cuerpos de aguas metales pesados causando graves daños al ecosistema. Un sistema de tratamiento con zeolitas y después con el jacinto de agua (Eichhornia crassipes) es económico, fácil de conseguir y ofrece resultados eficientes. También este material vegetal se puede convertir en la energía para cualquier tipo de industria.

\section{REFERENCIAS}

Atehortúa, E. \& Gartner, C. (2003). Preliminary studies of eichhornia crassipes dry biomass for lead and chromium removal from waters. Revista Colombiana de Materiales, 5.

Alvarado, A. \& Guédez, Merú. (2008). Arsenic removal from waters by bioremediation with the aquatic plants Water Hyacinth (Eichhornia crassipes) and Lesser Duckweed (Lemna minor). International Journal of Phytoremediation, 16(12).

Acevedo, D., Builes, S., Ordóñez, C. \& López, I. (2011). Evaluación de la eficiencia de una batería de filtros empacados en zeolita en la remoción de metales pesados presentes en un licor mixto bajo condiciones de laboratorio. Revista Ingenierías Universidad de Medellín, 10(18), 31-42.

Arenas, A., Merú, M. \& Gosmyr, T. (2011). Evaluation of the plant lemna minor for the bioremediation of water contaminated with mercury. Avances en Ciencias e Ingeniería, 2(3), 1-11.

Asgari, G., Ramavandi, B., Rasuli, L. \& Ahmadi, M. (2013). Adsorption from aqueous solution using a surfactant-modified Iranian zeolite: characterization, optimization, and kinetic approach. Desalination and Water Treatment, 51(31-33).

Aswathy, U., Rajeev, K. \& Sukumaran, G. (2010, January). Bio-ethanol from water hyacinth biomass: An evaluation of enzymatic saccharification strategy. Renewable Energy, 37(1), 109-116.

Bhattacharya, K. (2010). Water hyacinth as a potential biofuel crop. Electron J Environ Agric Food Chem, 9(1), 112-122.

Blanco, F., Ortega, H. \& Batista, N. (2014, mayo). Remoción de plomo (II) en vidrio volcánico y propuesta de adsorbido por etapas. Revista internacional de contaminación ambiental. Rev. Int. Contam. Ambient, 30(2).

Carrión, C., Ponce de León, S., Cram, I., Sommer, Hernández, M. \& Vanegas, C. (2012). Aprovechamiento potencial del lirio acuático (Eichhornia crassipes) en Xochimilco para fitorremediación de metales. Revista Agrociencia.

Chuang, Y.S., Lay, C.H., Sen, B., Chen, C.C., Gopalakrishnan, K. \& Wu, J.H. (2012, October). Biohydrogen and biomethane from water hyacinth (Eichhornia crassipes). International Journal of Hydrogen Energy, 36(21), 14195-14203. 
Chisutia, W. \& Mmari, O. (2014). Adsorption of Congo Red Dye from Aqueous Solutions Using Roots of Eichhornia crassipes: Kinetic and Equilibrium Studies. Energy Procedia. 50, 862-869.

Chutia P., S Kato, Kojima T., Satokawa S. (2009) La adsorción de As (V) en zeolitas naturales modificados tensioactivos. J Hazard Mater 162 (1): 204-211

Covarrubias, C., García, R., Yánez, J. \& Arriagada, R. (2008, August). Preparation of CPB-modified FAU zeolite for the removal of tannery wastewater contaminants. Journal of Porous Materials, 15(4), 491-498.

Du, G. (2012, June 30). Retention and transport through $\mathrm{Fe}$ (III)-coated natural zeolite. J. Hazard. Mater. Journal of Hazardous Materials. 221-222, 118-123.

Duarte, E., Olivero, J. \& Jaramillo, B. E. (2009). Remoción de cromo de aguas residuales de curtiembres usando quitosan obtenido de desechos de camarón. Scientia et Technica, 2(42).

Epstein, P. (2012). Weeds bring disease to the east African waterways. Lancet, 351(9102), 21, 577.

Gajera, A. \& Domadiya, P. (2013, Jul-Aug). Molecular mechanism of Trichoderma as bio-control agents against phytopathogen system. A review, $1(4)$.

Gómez, H. \& Pinzón, G. (2012). Análisis de la mitigación del impacto ambiental en el lago del parque la florida, por fitorremediación usando buchón de agua. Tesis de especialización Universidad Militar.

Gopal, B. (1987). Aquatic Plant Studies 1.Water Hyacinth. New York, USA: Elsevier Publishing.

Guocheng, L., Li, Z., Wei-Teh, J., Ackley, C. \& Fenske, N. (2014). Demarco removal of Cr (VI) from water using $\mathrm{Fe}$ (II)-modified natural zeolite. Chem. Eng. Chemical Engineering Research and Design (Impact Factor: 1.93), 92(2), 384390. DOI: 10.1016/j.cherd.2013.08.003
Han, Y., Shan, W., Ming, W., Nora, F. Y. T. (2008, October). Effects of anion species and concentration on the removal of $\mathrm{Cr}$ (VI) by a microalgal isolate, Chlorella min-iata. Journal of Hazardous Materials. 158(2-3), 615-620.

Higuera, O., Escalante, H. \& Laverde, D. (2005). Reducción del cromo contenido en efluentes líquidos de la industria del cuero, mediante un proceso adsorción - desorción con algas marinas. Scientia et Technica, 11(29).

Higuera, O., Arroyave, J. \& Flórez, L. (2008). Diseño de un biofiltro para reducir el índice de contaminación por cromo generado en las industrias del curtido de cueros. Falta el nombre de la revista, (160), 107-119.

Hossain, R., Chowdhury, M.K. \& Yeasmin, M. (2010). Production of ethanol using yeast isolates on water hyacinth and azolla. Bangladesh J. International Science Index. 7(1).

Hui, K., Chao, C. \& Kot, S. (2005). Removal of mixed heavy metal ions in wastewater by zeolite $4 \mathrm{~A}$ and residual products from recycled coal fly ash. J. Hazard. Mater. Journal of Hazardous Materials (Impact Factor: 4.33). 127(1-3), 89-101.

Lenka, M., Kamal, K. P. \& Brahma, B. (1990). Studies on the ability of water hyacinth (Eichhornia crassipes) to bioconcentrate and biomonitor aquatic mercury. Environmental Pollution, 66(1), 1-101.

Leyva, R., Azuara, P., Díaz, F. \& Guerrero, C. (2008, Nov). Adsorption of chromium (VI) from an aqueous solution on a surfactant-modified zeolite. Colloids and Surfaces A: Physicochemical and Engineering Aspects. 330(1), 35-41.

Ikhtyar, O. \& Shmsuzzamana, F. (1984). Kinetics of gas formation from water hyacinth biomass. In Proceedings of the international conference on water hyacinth, Hyderabad, India. p. 475-85.

Kasturiarachchi, J. C. (2014). Removal of nutrients (N and $\mathrm{P}$ ) and heavy metals (Fe, $\mathrm{Al}, \mathrm{Mn}$ and $\mathrm{Ni}$ ) from industrial wastewaters by phytoremedia- 
tion using water hyacinth (Eichhornia crassipes) under different nutritional conditions.

Katsou, E., Simos, M., Myrto, T., Haralambous, K. J. \& Loizidou, M. (2011). Tratamiento de aguas residuales contaminadas con Zinc y Plomo a través de zeolitas. J. Hazard. Mater., vol. 252-253, pp. 428-461.

Kumar, A. \& Ghosh, S. (2009). Bioconversion of lignocellulosic fraction of water-hyacinth (Eichhornia crassipes) hemicellulose acid hydrolysate to ethanol by Pichia stipites. Bangladesh J. Bot. 26: $1983-85$.

Kumar, V., Motohide, M. \& Michihiro, M. (2008). Sorption properties of the activated carbon-zeolite composite prepared from coal fly ash for $\mathrm{Ni} 2+$, $\mathrm{Cu} 2+, \mathrm{Cd} 2+$ and $\mathrm{Pb} 2+$. Bangladesh J. Bot. 26: 1983-85.

Magriotis, Z., Paulo, V. \& Leal, A. (2014). Treatment through zeolites etheramines. Applied Clay Science 91-92, 55-62.

Margeta, K., Zabukovec, N., Siljeg, M. \& Farkas, A. (2013). In water treat. (Elshorbagy, WInTech). Retrieved from http://www.intechopen.com/ books/water-treatment/natural-zeolites-in-water-treatment-how-effective-is-their-use.

Martínez, C., Torres, L. M., \& de la Cruz, R. F. G. (2013). Evaluación de la cinética de adsorción de zn2+ $\mathrm{y} \mathrm{cd} 2+$ a partir de soluciones unitarias y binarias por raíces de eichhornia crassipes y typha latifolia. Avances en Ciencias e Ingeniería, 4(2), 1-14.

Mishima, D., Kuniki, M., Sei, S., Ike, M. \& Fujita, M. (2008). Ethanol production from candidate energy crops: Water hyacinth (Eichhornia crassipes) and water lettuce (Pistia stratiotes L.). Bioreosur Tecnhol. 99, 2495-2500.

Pellón, A., Rodríguez, M. \& González, O. (2011). Remoción de cromo mediante el uso de un bioreactor utilizando scenedesmus obliquus inmovilizado. Ingeniería Hidráulica y Ambiental, 32, (334335), 161-166.
Pitcher, S. K., Slade, R. C. T. \& Ward, N. I. (2004, Dec.). Heavy metal removal from motorway stormwater using zeolites. Sci Total Environ., 1(334335), 161-166.

Poddar, K., Mandal, L. \& Banerjee, G.C. (1991). Studies on water hyacinth (Eichhornia crassipes), chemical composition of the plant and water from different. C.T.F Cienc. Tecnol. Futuro, 5(2).

Porous, D., Siregar, H. \& Salem, F. (2012). Studies on the uses of water hyacinth as biogas energy resource in the dam of curag (west java). In Proceedings of the international conference on water hyacinth, Hyderabad. chemical composition of the plant and water from different habitats. Indian Veterinary Journal, 68(9), 833-837.

Qiu, W. \& Ying, Z. (2009, January 1st). Removal of lead, copper, nickel, cobalt, and zinc from water by a cancrinite-type zeolite synthesized from fly ash. Chemical Engineering Journal, 145(3), 483-488.

Rodríguez, A. (2013). Estudio de la cepa Strepcoccus y Kingella en remoción de metales pesados en aguas industriales. Estudio de Caso.

Ríos, A., Vargas, F. \& Cuchimaque, L. (2013, marzo). Remoción de $\mathrm{Fe}$ y Mn en aguas naturales por adsorción-oxidación sobre clinoptilolita. Rev. Fac. Ing. Univ. Antioquia, (66), 24-44.

Saraswat, J. (2010, April 28). Heavy metal adsorption from aqueous solution using Eichhornia crassipes dead biomass. International Journal of Mineral Processing, 94(3-4), 203-206.

Torres, M. (2009). Estudio del aprovechamiento del lechuguin, Eichhornia crassipes, del embalse de la represa Daniel Palacios como biosorbente de metales pesados en el tratamiento de aguas residuales. Tesis de Grado. Universidad Militar nueva Granada. Bogota, DC.

Velarde, H., Zavaleta, A. \& Aguilar, Q. (2013). Estudio de la absorción del ion cromo VI con jacinto de agua (Eichhornia crassipes). En Segundo en- 
cuentro de investigadores. Universidad Nacional de Trujillo.

Veranes, O., Pellón, A., Espinosa, M. \& Oña, A. (2009, enero-abril). Estudio del comportamiento de la microalga scenedesmus obliquus para la precipitación de cromo en albañal sintético. Revista Cubana de Química, 24(1).

Wanga, S. \& Peng, Y. (2010, January). Natural zeolites as effective adsorbents in water and wastewater treatment. Chem. Chemical Engineering Journal. 156(1), 11-24.

Wanga, S. \& Yuelian, P. (2010). Natural zeolites as effective adsorbents in water and wastewater treatment. Chemical Engineering Journal 156, 11.
Xiaosen, L., Songlin, L., Zhongyuan, N., Diannan, L. \& Zheng, L. (2013, Nov.). Adsorption, concentration, and recovery of aqueous heavy metal ions with the root powder of Eichhornia crassipes. Ecological Engineering, 60, 160-166

Yubin, T., Qunjie, X. \& Yulin, M. (2015). Study of Uranium Accumulation Mechanism and Physiological Responses of Eichhornia crassipes and Pistia stratiote. Environmental Protection and Resource Utilization IV. Advanced Materials Research, 1073-1076.

Zimmels, F. M. (2005).Application of Eichhornia crassipes and Pistia stratiotes for treatment of urban sewage in Israel. Journal of Environmental Management (Impact Factor: 3.19) 